\title{
CHROMOSOME BEHAVIOUR IN SPECIES HYBRIDS WITH TRITICUM TIMOPHEEVI
}

\author{
LEO SACHS \\ Plant Breeding Institute, Cambridge \\ John Innes Horticultural Institution, Bayfordbury, Hertford, Herts
}

Received 28.xi.5I

\section{INTRODUCTION}

THE 28 chromosome wheat species Triticum timopheevi has attracted interest on account of its reported cytological behaviour and its application to the genome concept. Lilienfeld and Kihara (I934) had examined the $F_{1}$ hybrids between tetraploid wheat species. They had found that the hybrids involving timopheevi differed from the rest, were normally sterile, and had poor chromosome pairing. On the basis of chromosome pairing in hybrids they designate the chromosome sets of timopheevi by the formula $\mathrm{AG}$ in contrast to the $\mathrm{AB}$ formula given to the chromosome sets of the other tetraploid species. In their classification of the genus Triticum, the species timopheevi is also placed in a section of its own thus suggesting a different origin. This suggestion is followed by Sears (1948). Kostoff (1937) obtained similar results to those of Lilienfeld and Kihara, but he interprets them as showing " partial homology" (as measured by metaphase chromosome pairing in hybrids) between the chromosome sets of timopheevi and of the other tetraploid species. Kostoff therefore suggests for timopheevi the formula $A \beta$.

In view of this reported behaviour of timopheevi, it was decided to investigate hybrids with this species using both $F_{1}$ 's and a range of colchicine derived amphiploids. The results obtained do not support either the genome concept or the suggested mode of origin of timopheevi.

\section{MATERIAL AND TECHNIQUES}

The following parental species and varieties have been used in this investigation :-

$2^{n}=14$ chromosomes

T. monococcum L. var. vulgare Körn.

$2^{n}=28$ chromosomes

T. dicoccoides (a) Körn. var. nudiglumis Nabalek

$T$. dicoccoides (b) Körn. var. kotschyanum Perc.

$T$. dicoccum Schübl. var. farrum Körn.

T. polonicum L. var. levissimum Körn.

T. turgidum L. var. iodurum Körn.

T. turanicum Jacubz. $(=T$. orientale Perc. $)$ var. notabile Perc.

T. carthlicum Nevski. (= T. persicum Vav.)

T. timopheevi Zhuk var. typicum Zhuk. 
$T$. dicoccoides var. nudiglumis will be referred to as dicoccoides (a). Specimens of this variety were obtained from Mr J. B. Gillett (now at the Kew Herbarium, England) who had found the plants growing in Iraq, and following the botanical description of Nabalek (1929) they have been assigned to the variety nudiglumis. $T$. dicoccoides var. kotschyanum will be referred to as dicoccoides (b). Seeds of some of the $F_{1}$ hybrids and of the amphiploids were placed at my disposal by $\mathrm{Dr}$ G. D. H. Bell.

After a preliminary examination of meiosis on temporary acetocarmine smears, all cytological observations were made on Feulgen squashes. Anthers were fixed in $1: 3$ acetic alcohol and stained according to the Feulgen technique. Permanent preparations mounted in "Euparal" were made after the schedule outlined in Darlington and La Cour (1947, p. I 28). For a study of chromosome morphology in root tips, best results were obtained after pre-treatment for approximately three hours in a saturated solution of mono-bromo-naphthalene (O'Mara, 1948). This gave clearer chromosome figures than pre treatment with either low temperatures, paradichlorobenzene, chloral hydrate, or colchicine. Permanent preparations of root tips were also made by means of Feulgen squashes.

Unless otherwise stated, the figures on chromosome pairing are in each case based upon an analysis of 50 complete nuclei at the first metaphase of meiosis.

\section{CHROMOSOME BEHAVIOUR IN F SPECIES HYBRIDS}

Chromosome pairing in six 28 chromosome $F_{1}$ hybrids between timopheevi and other $4 x$ species of the genus Triticum is shown in table I. The first five of these hybrids show a reduction from the possible 14 bivalents per cell and from the 29.94 chiasmata per nucleus found in the species timopheevi. In addition :-

carthlicum $\times$ timopheevi $(4 x)$ showed an average of $6 \cdot 16$ I, 9.44 II, 0.96 III and 0.02 IV.

timopheevi $\times$ polonicum $(4 x)$ showed an average of $7.76 \mathrm{I}, 8.66 \mathrm{II}$, 0.92 III and 0.04 IV.

timopheevi $\times$ monococcum $(3 x)$ showed an average of $7 \cdot 80$ I, $6 \cdot 09$ II and 0.34 III.

The anthers did not dehisce in any of these $F_{1}$ hybrids and no seed was set. All these $F_{1}$ hybrids were therefore completely sterile.

Although previous workers have presented no adequate data for chiasma frequencies, these results broadly agree with those obtained by Lilienfeld and Kihara (1934) and Kostoff (1937). The $3^{x}$ hybrid shows that the chromosome pairing in a hybrid between timopheevi and a diploid species of Triticum is not very different from some other $4^{x} \times 2 x$ species hybrids in Triticum. The $4^{x}$ hybrids show that there is a decreased chromosome pairing in hybrids between timopheevi and 
other $4^{x}$ species of Triticum, but there is no hybrid which even approaches an average of 14 univalents per cell. There is therefore always some chromosome pairing between the two chromosome sets of timopheevi and those of the other species of Triticum.

Chromosome pairing in the $4^{x} \mathrm{~F}_{1}$ hybrid diococcoides $(\mathrm{a}) \times$ timopheevi (table I) is, however, different from the chromosome pairing found in all the other $4^{x} \mathrm{~F}_{1}$ hybrids involving timopheevi. This hybrid shows 13.90 bivalents per cell and 28.54 chiasmata per nucleus. No chromosome bridges were found at anaphase and the tetrads appeared to be normal. Despite this apparently normal meiosis the anthers did not dehisce and under the glasshouse conditions of 1949 the hybrid plants were completely sterile.

TABLE I

Chromosomc pairing in $F_{1}$ hybrids of $\mathrm{T}$. timopheevi with other $4 \mathrm{X}$ species

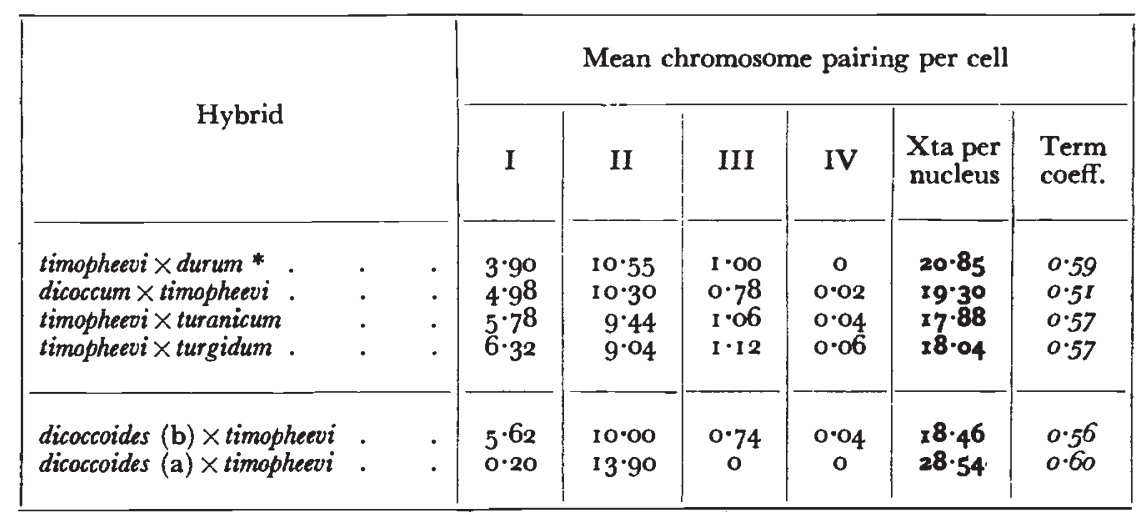

$*=20$ cells; in all other hybrids 50 cells were analysed.

$\mathrm{Xta}=$ chiasmata. Term. coeff. $=$ terminalisation coefficient.

In view of this result, hybrids were again made in 1949 using dicoccoides (a) on this occasion as both male and female parent. These hybrid plants were grown in two different lots in two open fields surrounded by many other fertile plants in order to ensure an ample opportunity for their pollination by foreign pollen.

Twenty-six $F_{1}$ plants were grown in 1950 and independently of whether dicoccoides (a) had been used as male or female parent the meiotic investigations confirmed the results which had been obtained in the previous year. Meiosis in the anthers was apparently normal in all cases, but none of the anthers dehisced. Degeneration of the gametes must therefore have occurred after the tetrad stage. From artificially opened mature anthers, a count of 500 pollen grains stained in aceto-carmine gave an estimate of 5 per cent. good pollen. Under the conditions of open pollination prevailing in the field in 1950 there was however some seed set. This was probably due to the greater viability of female gametes. 


\section{CHROMOSOME BEHAVIOUR IN AMPHIPLOIDS}

Further evidence on the chromosome behaviour in hybrids of timopheevi can be obtained by a study of colchicine-derived amphiploids from the $4^{x} \mathrm{~F}_{1}$ hybrids. The chromosome pairing of six amphiploids (derived from the six $F_{1}$ hybrids described in table $I$ ) is given in table 2.

Meiosis was studied in the first generation after colchicine treatment of the $\mathrm{F}_{1}$ hybrid plants. Of particular interest is the 56 chromosome amphiploid dicoccoides (a) $\times$ timopheevi which had shown 13.90 bivalents in its 28 chromosome sterile $F_{1}$ hybrid. In contrast to its $4 x F_{1}$ hybrid,

TABLE 2

Chromosome pniring in $8 \mathrm{x}$ amphiploids of $\mathrm{T}$. timopheevi

\begin{tabular}{|c|c|c|c|c|c|c|c|}
\hline \multirow{2}{*}{ Amphiploid } & \multicolumn{7}{|c|}{ Mean chromosome pairing per cell } \\
\hline & $\mathbf{I}$ & II & III & IV & $\mathrm{V} / \mathrm{VI}$ & $\begin{array}{l}\text { Xta per } \\
\text { nucleus }\end{array}$ & $\begin{array}{l}\text { Term } \\
\text { coeff. }\end{array}$ \\
\hline $\begin{array}{l}\text { timopheevi } \times \text { durum } \\
\text { dicoccum } \times \text { timopheevi } \\
\text { timopheevi } \times \text { turanicum } \\
\text { timopheevi } \times \text { turgidum }\end{array}$ & $\begin{array}{l}I \cdot 70 \\
I \cdot 04 \\
0 \cdot 86 \\
0 \cdot 92\end{array}$ & $\begin{array}{l}23 \cdot 18 \\
23 \cdot 94 \\
23 \cdot 50 \\
24 \cdot 06\end{array}$ & $\begin{array}{l}0 \cdot 38 \\
0 \cdot 24 \\
0.26 \\
0 \cdot 18\end{array}$ & $\begin{array}{l}\mathrm{I} \cdot 70 \\
1 \cdot 56 \\
\mathrm{I} \cdot 84 \\
\mathrm{I} \cdot 58\end{array}$ & $\begin{array}{r}0 \\
0 \cdot 02 \\
0 \\
0 \cdot 02\end{array}$ & $\begin{array}{l}54 \cdot 70 \\
50 \cdot 74 \\
54 \cdot 22 \\
55 \cdot 76\end{array}$ & $\begin{array}{l}0.57 \\
0.60 \\
0.56 \\
0.59\end{array}$ \\
\hline $\begin{array}{l}\text { dicoccoides (b) } \times \text { timopheevi } \\
\text { dicoccoides (a) } \times \text { timopheevi }\end{array}$ & $\begin{array}{l}I \cdot 48 \\
1 \cdot 47\end{array}$ & $\begin{array}{l}23 \cdot 28 \\
21 \cdot 10\end{array}$ & $\begin{array}{l}0 \cdot 36 \\
0 \cdot 47\end{array}$ & $\begin{array}{l}1 \cdot 72 \\
2 \cdot 73\end{array}$ & $\begin{array}{l}\mathbf{0} \\
\mathbf{o}\end{array}$ & $\begin{array}{l}5 x \cdot 44 \\
5 x \cdot 44\end{array}$ & $\begin{array}{l}0.64 \\
0.64\end{array}$ \\
\hline
\end{tabular}

* $=30$ cells $:$ in all other amphiploids 50 cells were analysed.

this $8 x$ amphiploid did show dehiscence of the anthers. Two plants were examined for male and female fertility in $195^{\circ}$ :-

(i) A count of Iooo pollen grains stained in aceto-carmine gave an average of 75 per cent. good pollen.

(ii) Primary florets, open pollinated, of 125 spikelets showed a range of seed set per spike from 29 to 72 per cent. giving an average of 55 per cent. seed set.

The $8 x$ amphiploid was therefore (like all the others) both male and female fertile.

The chiasma frequency per nucleus in the amphiploid dicoccoides (a) $\times$ timopheevi with $2 \cdot 73$ IV $(54.8$ per cent. of which formed rings) and 0.47 III, is the same as the chiasma frequency per nucleus in the amphiploid dicoccoides (b) $\times$ timopheevi with $\mathrm{I} \cdot 72$ IV $(54 \cdot 7$ per cent. of which formed rings) and 0.36 III. Multivalent formation, therefore, did not influence the chiasma frequency per nucleus in these two amphiploids. In common with the other hybrids, multivalent formation in the amphiploid dicoccoides (a) $\times$ timopheevi is lower than bivalent formation in the $F_{1}$ hybrid. This is probably due to differential affinity ( $c f$. Darlington, 1937). 


\section{CHROMOSOMAL STERILITY IN SPECIES HYBRIDS}

Dobzhansky (I94I) distinguishes between genic and chromosomal sterility in species hybrids. He points out that the principal source of evidence for the existence of chromosomal sterility in an $F_{1}$ hybrid is the behaviour of its allopolyploid. The production of a fertile amphiploid from the sterile $F_{1}$ dicoccoides $(a) \times$ timopheevi would thus indicate that the $F_{1}$ sterility is chromosomal, and the same applies to the other five amphiploids described in the previous section. The $F_{1}$ sterility of these other five amphiploids is, however, at least partly due to the formation of univalents and the subsequent abnormalities in meiosis, whereas the $F_{1}$ hybrid dicoccoides $(a) \times$ timopheevi was sterile despite an apparently normal meiosis.

TABLE 3

Chiasma frequency of parents, $F_{1}$ hybrid, and amphiploid $T$. dicoccoides $(a) \times T$. timopheevi

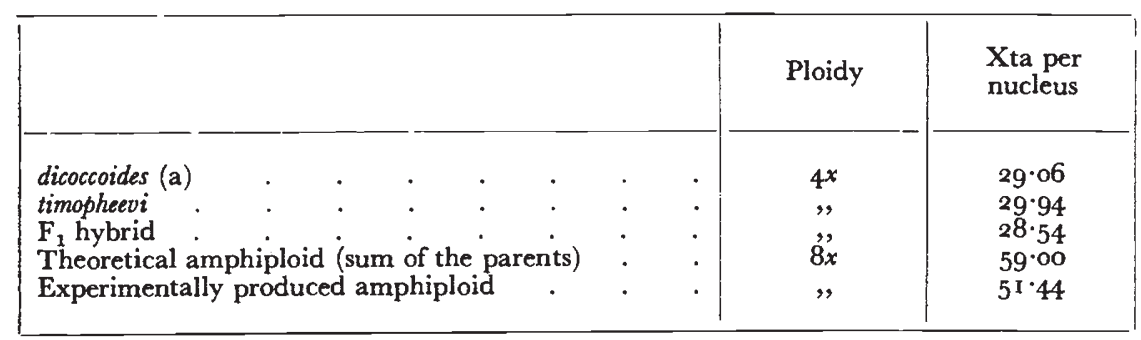

50 cells analysed in each species and hybrid.

Table 3 shows the chiasma frequency of the parent species, $F_{1}$ hybrid, and amphiploid dicoccoides (a) $\times$ timopheevi. This shows that the chiasma frequency of the $F_{1}$ hybrid was almost as high as that of the parent dicoccoides (a) and only slightly less than the chiasma frequency of the parent timopheevi. The observed amphiploid chiasma frequency was lower than the chiasma frequency derived from the sum of the two parent species. The ratio of observed to calculated chiasma frequency is 0.87 for chiasmata per nucleus. This ratio is comparable to that found in the other Triticum amphiploids, and in Primula kewensis (Upcott, I939). The multivalent frequency in Primula kewensis is lower however than that found in the amphiploid dicoccoides (a) $\times$ timopheevi.

The behaviour of Primula kewensis has been explained by Darlington (1937) followed by Dobzhansky (I94I) and Stebbbins (1950). It is assumed that the chromosomes of the two parent species are sufficiently alike to ensure chromosome pairing in the $F_{1}$ hybrid, but that non-homologous segments are involved in this apparently normal chromosome pairing. The gametes produced by the $F_{1}$ hybrid will then be inviable because recombination between the chromosomes of the two parent species has produced inviable combinations of genic 
material. Pairing and segregation in the amphiploid produced by somatic doubling would, however, tend to produce gametes containing one complete set of chromosomes derived from one parent and one complete set of chromosomes derived from the other parent. These gametes would then be viable.

The type of chromosomal sterility in $F_{1}$ hybrids, which is brought about by chromosomal differences which do not materially affect metaphase chromosome pairing at meiosis, has been called cryptic structural hybridity by Stebbins (1945, I950). The high degree of sterility in the $F_{1}$ hybrids, such as timopheevi $\times$ durum with a mean of 3.90 I per cell and in $F_{1}$ hybrids between timopheevi and hexaploid species of Triticum, may also be partly due to cryptic structural hybridity.

TABLE 4

Chromosome pairing in $4 \times F_{1}$ hybrids of $\mathrm{T}$. timopheevi,

$\mathrm{T}$. dicoccoides $(a)$ and $\mathrm{T}$. dicoccoides $(b)$

\begin{tabular}{|c|c|c|c|c|c|c|}
\hline \multirow{2}{*}{ Hybrid } & \multicolumn{6}{|c|}{ Mean chromosome pairing per cell } \\
\hline & I & II & III & IV & $\begin{array}{l}\text { Xta per } \\
\text { nucleus }\end{array}$ & $\begin{array}{l}\text { Term } \\
\text { coeff. }\end{array}$ \\
\hline dicoccoides (a) $\times$ timopheevi & 0.20 & $13 \cdot 90$ & o & o & $28 \cdot 54$ & $0 \cdot 60$ \\
\hline dicoccoides (b) $\times$ timopheevi & $5 \cdot 62$ & $10 \cdot 00$ & $0 \cdot 74$ & $0 \cdot 04$ & $18 \cdot 46$ & 0.56 \\
\hline dicoccoides (a) $\times$ dicoccoides $(\mathrm{b})$ & $10 \cdot 16$ & $8 \cdot 02$ & $0 \cdot 60$ & o & $\times 3 \cdot 3^{6}$ & 0.52 \\
\hline
\end{tabular}

50 cells analysed in each hybrid.

Further evidence on these questions can be obtained indirectly. If chromosome pairing in the $F_{1}$ hybrid dicoccoides (a) $\times$ timopheevi involves non-homologous segments (as is indicated by the evidence presented above) these two parent forms may show a difference in chromosome pairing in other hybrid combinations. The amount of structural similarity sufficient to ensure good chromosome pairing between the two parent forms in the hybrid dicoccoides (a) $\times$ timopheevi may not be sufficient to ensure equal chromosome pairings when each of these parents is involved in other hybrid combinations. That this is actually the case is seen from the data in table 4 .

With dicoccoides (b) as one of the pairents, only 5.62 chromosomes remain unpaired in hybrids with timopheevi whereas $10 \cdot 16$ chromosomes remain unpaired in hybrids with dicoccoides (a). The possibility of genotypic (as opposed to structural) control of chromosome pairing producing the differences found in these two hybrids cannot be entirely ruled out. But these data on chromosome pairing can also be explained if some of the chromosomes of dicoccoides (a) and timopheevi are sufficiently unlike to give differences of pairing in their hybrids 
with dicoccoides (b). These differences in pairing and the sterility of the $\mathrm{F}_{1}$ hybrid dicoccoides (a) $\times$ timopheevi can thus both be explained by the existence of structural differences between the chromosomes of all three stocks.

\section{THE GENOME CONCEPT AND THE ORIGIN OF TETRAPLOID TRITICUM SPECIES}

Largely as a result of Kihara and his co-workers (Kihara, I937, 1949) the concept of genome formulæ has become prominent within the sub-tribe Triticine. Metaphase chromosome pairing in hybrids is taken as an indication of chromosome homology and each chromosome set (called a genome) is given a specific formula. Some of the data from chromosome pairing can be used for the interpretation of phylogenetic relationships in conjunction with external plant morphology. Thus Kihara (1944) suggested that 14 chromosomes belonging to the chromosome sets of the $6 x$ species of Triticum have been derived from the I4 chromosome species Aegilops squarrosa. This suggestion was experimentally confirmed by the actual synthesis of plants resembling $6 x$ species of Triticum (MacFadden and Sears, I 946 ; Kihara and Lilienfeld, I 949).

Darlington (1937) has, however, stressed the undesirability of basing phylogenetic conclusions on chromosome pairing in hybrids. Dobzhansky (194I) has also pointed out that what is actually being studied is the chromosome pairing which is mainly determined by the gross structural arrangement of the chromosomes. The term genome implies that chromosome sets with the same genome formula contain the same gene complexes. There is, however, no evidence to suggest that the amount of genic differentiation is always proportional to the amount of structural differentiation which is able to influence chromosome pairing at the first metaphase of meiosis. Kihara (1937) has himself pointed out that there is no distinct dividing line between what is called a homologous and what is called a non-homologous genome, the one gradually merges into the other.

In most cases, metaphase chromosome pairing and chiasma formation in hybrids is assumed as being determined by the gross structural arrangements of the chromosomes. Phylogenetic speculations based only on the criterion of metaphase chromosome pairing can, however, lead to erroneous conclusions. The formulæ given to the chromosome sets of the $4 x$ species of Triticum are a case in point.

The AG formula suggested by Lilienfeld and Kihara (1934) for timopheevi is also applied by Sears (1948) to the chromosome sets of armeniacum, and it is further suggested that the $G$ chromosome set of both armeniacum and timopheevi is probably phylogenetically derived from a different species than the $B$ chromosome set of the other tetraploids. The evidence for suggesting the formula $G$ for one of the chromosome sets of timopheevi is based on the reduction of chromosome 
pairing in $4^{x}$ species hybrids involving timopheevi. The evidence for assigning the formula $G$ to one of the chromosome sets of armeniacum is based on the data of Svetozarova (1939) that there is good chromosome pairing and apparently normal tetrads in the hybrid timopheevi $\times$ armeniacum. The data of Svetozarova (1939) however, also shows that despite an apparently almost normal meiosis the hybrid was sterile. The sterility in $\mathrm{F}_{1}$ timopheevi $\times$ armeniacum may also be due to cryptic structural hybridity.

The chromosome behaviour of $F_{1}$ timopheevi $\times$ armeniacum is thus similar to that in $F_{1}$ timopheevi $\times$ dicoccoides (a) and in both cases meiosis would involve the pairing of chromosomes with non-homologous segments.

If one now follows the principles which are accepted in the giving of formulæ on the basis of metaphase chromosome pairing in hybrids, dicoccoides (a) would have to be given the formula AG in common with timopheevi and armeniacum. One would then be in a position either of having to elevate dicoccoides (a) into a new species (for which there is no justification) or of designating the variety dicoccoides (a) as $\mathrm{AG}$ and the variety dicoccoides (b) as $\mathrm{AB}$. If the two varieties are designated as $A B$ and $A G$ one would then have to postulate that the tetraploid chromosome sets of these two varieties of dicoccoides are phylogenetically derived from two different species, which is highly improbable. This, therefore, shows the misleading conclusions that can be obtained when basing phylogenetic relationships only upon the amount of metaphase chromosome pairing at meiosis.

Considerable divergence in chromosome structure, as analysed by pairing at meiosis, has been found within the species dicoccoides. There is thus no reason to suppose that timopheevi and armeniacum may not also have diverged in the same way from an original $4 x$ prototype. An examination of the chromosome morphology of timopheevi shows that, in common with the other $4 x$ species of Triticum, it has at mitosis chromosomes with median or sub-median centromeres and four chromosomes with large satellites. It is not possible to identify with certainty all the individual chromosomes and chromosome measurements can differ appreciably in various varieties of the same species of Triticum ( $c f$. Camara, I 947). The basic idiogram regarding the presence of median or submedian centromeres and the number of chromosomes with satellites is, however, the same in timopheevi as in the other $4^{x}$ species. This evidence also conflicts with the suggestion that the tetraploid chromosome set of timopheevi has been phylogenetically derived from another species than the tetraploid chromosome set of e.g. dicoccoides.

The specimens designated as dicoccoides (a) are not morphologically identical with armeniacum. Armeniacum, among other characteristics, does not occur together with Hordeum spontaneum; it has no varieties with pubescent spikes; and it was found in Armenia and at Nakhichevan in Transcaucasia (Jukubziner, I932 ; Tumanjan, I 934 ; 
Makushina, 1938). Mr J. B. Gillett has informed me that there was considerable morphological variation in the specimens of dicoccoides which he found in Iraq. The plants were growing on the northern slopes of Handren Dagh above Jundiyan village a few miles east of Rowanduz. There were pubescent and glabrous forms of various colours and noticeable differences in the size of spike and general vigour. In contrast to armeniacum in Transcaucasia, the dicoccoides in Iraq was found growing together with Hordeum spontaneum. The considerable morphological range of dicoccoides in Iraq may on further cytological examination also show a range of chromosome differentiation from the Syrio-palestinicum types such as dicoccoides (b) to the cytologically differentiated types such as dicoccoides (a) and timopheevi. There is thus nothing improbable in the suggestion that all the 28 chromosome species of Triticum have originated from a common 28 chromosome prototype. The various stages of cytological differentiation may, in fact, still be in existence in Iraq.

\section{SUMMARY}

I. Two types of $T$. dicoccoides (a) and (b), have been found to differ in their chromsome structure. These types show that all the cytological differentiation among the $4^{x}$ species of Triticum can be found within the species dicoccoides.

2. All the cytologically different $4^{x}$ species of Triticum could thus have been derived from an original 28 chromosome prototype.

3. Tetraploid species hybrids with $T$. timopheevi show sterility presumably owing to recombination between chromosomes containing non-homologous segments.

4. Metaphase chromosome pairing in hybrids is not sufficient to assign distinct genome formulæ for the properties of different chromosome sets in various species.

Acknowledgment.-This work was mainly carried out at the Plant Breeding Institute, Cambridge, and I am indebted to Dr G. D. H. Bell for his constant irterest.

\section{REFERENCES}

camara, A. 1947. Cromosomas somaticos de Triticum turgidum. Agron. Lusitana, 9, I 7 I - I 80 .

DarLington, C. D. 1937. Recent Advances in Cytology. 2nd Ed. London : Churchill. DARLington, C. D., AND LA COUR, L. F. 1947. The Handling of Chromosomes. 2nd Ed. London: Allen \& Unwin.

Dobzhansky, T. r94I. Genetics and the Origin of Species. 2nd Ed. New York : Columbia University Press.

JAKUBZINER, M. 1932. Contribution to the knowledge of wild wheats in Transcaucasia. Bul. Appl. Bot. Gcn. \& Pl. Br., Series 5, No. 1, I47-198.

kıhara, H. I 937 . Genomanalyse bei Triticum und Aegilops VII Kurze Übersicht über die Ergebnisse der Jahre 1934-36. Kyoto Univ. Col. Agr. Mem., No. 4r, I 6 I.

KIHARA, H. I944. Discovery of the DD analyser, one of the ancestors of Vulgare wheats. Agr. \& Hort., 19, 889-89o. 
KinARA, H. 1949. Genomanalyse bei Triticum und Aegilops IX Systematischer Aufbau der Gattung Aegilops auf Genomanalytischer Grundlage. Cytologia, $14,135^{-1} 44$.

kimara, H., AND lilienfeld, F. 1949. A new synthesised $6 x$ wheat. Proc. 8th Int. Genet. Congr. (Stockholm), pp. 307-319.

коstofF, D. 1937. Chromosome behaviour in Triticum hybrids and allied genera I interspecific hybrids with Triticum timopheevi. Proc. Indian Acad. Sci., 5, 231-236. Lilienfeld, F., AND kihara, H. 1934. Genomanalyse bei Triticum und Aegilops V Triticum timopheevi Zhuk. Cytologia, 6, 87-122.

MACFADDEN, E. S., AND SEARS, E. R. 1946. The origin of Triticum spelta and its free threshing hexaploid relatives. 7. Hered., 37, 81-89, 107-1 16.

MAKUSHINA, E. N. 1938. A new species of wheat Triticum armeniacum (Jakubz.) sp. n. C.R. Acad. Sci. U.S.S.R.., 21, 345-348.

Nabalex, F. 1929. Inter turco-persicum pars. V. Publ. Fac. Sci. Masaryk Univ. Brno., $111,27-28$.

o'mARA, J. G 1948. Acetic acid methods for chromosome studies at prophase and metaphase in meristems. Stain Tech., 23, 201-204.

SEARS, E. R. 1948. The cytology and genetics of the wheats and their relatives. Adv. Genet., 2, 239-270.

STEBBINS, G. L. JR. 1945. The cytological analysis of species hybrids II. Bot. Rev., $11,463-486$.

STEBbins, G. L. JR. 1950. Variation and Evolution in Plants. New York : Columbia University Press. London: Oxford University Press.

sVetozarova, v. v. 1939. Second genome of Triticum timopheevi Zhuk. C.R. Acad. Sci. U.S.S.R., 23, 473-477.

tumanyan, M. 1934. The botanical diversity of the wild wheats in Armenia. Bul. Appl. Bot. Gen. EO Pl. Br., Series 5, No. 2, 241-268.

upсотт, м. 1939. The nature of tetraploidy in Primula kewensis. 7. Genet., 39, 79-100. 


\section{Plate}

Microphotographs of pollen mother cells at Metaphase I.

Feuglen stained. $\times 1600$.

F1G. 1. $-4 x$ timopheevi $\times$ turgidum 5 I, IO II, I III.

F1G. 2.-4x dicoccoides (a) $\times$ timopheevi 14 II.

FIG. 3.-8x dicoccum $\times$ timopheevi 3 I, I9 II, I III, 3 IV. 


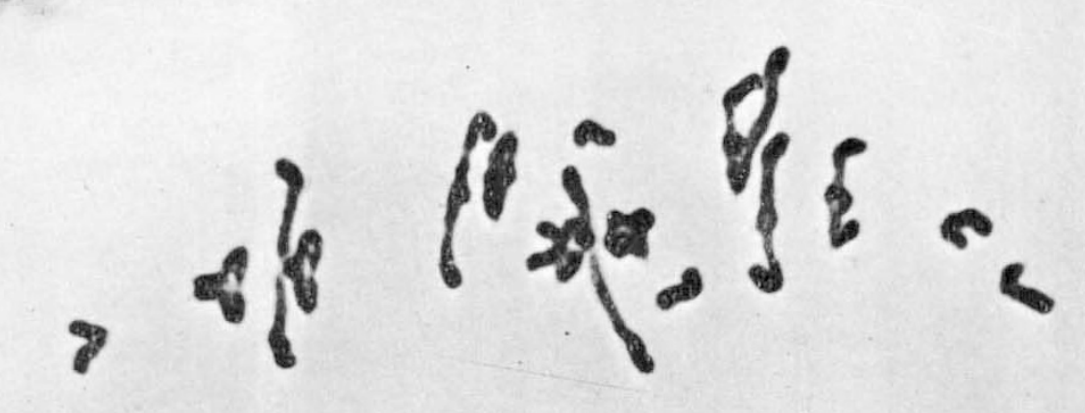

- gerte 10

$$
\text { व }
$$

